\title{
Aorta of young and middle-aged heterozygous familial hypercholesterolemia patients shows no functional or morphological impairment assessed by MRI
}

\author{
Sami Soljanlahti' \\ Taina Autti' \\ Alpo F Vuorio² \\ Pekka Keto' \\ Hannu Turtola ${ }^{3}$ \\ Kirsi Lauerma'
}

'Helsinki Medical Imaging Center, Helsinki University Central Hospital, Helsinki, Finland; 'Division of Internal Medicine, Department of Medicine, University of Helsinki, Helsinki, Finland; ${ }^{3}$ Department of Internal Medicine, North Karelia Central Hospital, Joensuu, Finland
Correspondence: Sami Soljanlahti Helsinki Medical Imaging Center, Helsinki University Central Hospital, PL 340, 00029 HUS, Finland Tel +358947174417

Fax +358947174404

Email sami.soljanlahti@hus.fi

\begin{abstract}
In familial hypercholesterolemia (FH) the level of LDL cholesterol is 2-3 times that of the normal population and leads to accelerated atherosclerosis. Improved care for risk factors has decreased cardiovascular mortality of these patients. We studied subclinical atherosclerotic changes with morphologic and functional aortic magnetic resonance imaging (MRI) in FH patients under the age of 50. 39 DNA test-verified heterozygous FH-North Karelia patients, aged 6-48, 28 of them treated with statins, and 25 healthy controls, aged 12 to 50, underwent aortic MRI, carotid ultrasound (US), and risk-factor assessment. No differences in any of the morphologic or functional aortic parameters appeared between patients and controls. Age and gender were independent predictors of the majority of the morphologic and functional measures. Carotid intima-media thickness assessed by US was greater in patients $(0.57 \mathrm{~mm} \pm 0.13 \mathrm{vs}$ $0.48 \pm 0.13 \mathrm{~mm}, \mathrm{p}=0.005)$ as was cholesterol-years score $(243 \pm 122$ vs $137 \pm 74, \mathrm{p}<0.001)$. Patients had thicker intima-media of the common carotid artery and higher cholesterol burden as indicated by their cholesterol-years score. Despite this, no differences existed in morphologic or functional aortic parameters assessed with MRI. The improved care of cardiovascular risk factors, especially statin treatment, may protect the aorta of FH patients. However, larger confirmatory studies are needed.
\end{abstract}

Keywords: MRI, ultrasound, atherosclerosis, aorta, familial hypercholesterolemia

\section{Introduction}

Familial hypercholesterolemia (FH) is a hereditary disease caused by a low-density lipoprotein (LDL) receptor defect, which results in impaired cholesterol uptake in the liver (Goldstein and Hobbs 2001). The heterozygous form of the disease leads to 2- to 3-fold higher blood cholesterol levels from birth compared with those of the normal population, which results in accelerated atherosclerosis. The prevalence of heterozygous $\mathrm{FH}$ is $1 / 500$. Atherosclerotic lesions of $\mathrm{FH}$ patients decrease with statin treatment (Smilde et al 2001), but patients still have higher (cardiovascular) mortality than does the normal population (Anonymous 1999). In the early 1990s the mean age of onset of coronary heart disease (CHD) was 42 and 48 years respectively for Finnish male and female FH patients with the North Karelia (FH-NK) mutation. Thirty percent of the patients over age 25 had CHD (Vuorio et al 1997). In contrast, in year 2000 the combined prevalence for acute myocardial infarction and angina pectoris in the 30 - to 64-year-old general Finnish population was $3.8 \%$ and $1.3 \%$ for men and women, respectively (Reunanen et al 2004).

In atherosclerosis inflammation, accumulating lipids and fibrosis change arterial morphology and elastic properties. The morphology in vascular MRI correlates well 
with histology (Clarke et al 2003) and elastic properties can be measured by magnetic resonance imaging (MRI) (Oliver and Webb 2003). A positive association exists in autopsy material between aortic and coronary atherosclerosis (Sternby 1968). Atherosclerotic lesions can be detected and characterized with MRI (Yuan and Kerwin 2004). In the 1990s signs of subclinical atherosclerosis appeared in aortic MRI of $40 \%$ of clinically healthy people age 60 , on average (Jaffer et al 2002).

The hypothesis of our study was, that despite the improved prognosis of $\mathrm{FH}$ patients due to developed risk factor treatment (statin treatment in particular), MRI of the aorta would reveal subclinical atherosclerosis. Our aim was to find out if functional and morphological parameters of the aorta differ in young symptomless FH patients and normal controls. To assure the clinical relevance of the MRI results, we compared them with intima-media thickness (IMT) and strain of common carotid artery (CCA) assessed by ultrasound (US), and with cardiovascular risk factors (blood cholesterol levels, age, blood pressure, and body mass index: BMI).

\section{Methods}

\section{Study participants}

The province of North Karelia in Finland has approximately 170,000 inhabitants and FH-NK mutation accounts for $90 \%$ of its familial hypercholesterolemia cases (Koivisto et al 1992). During a 1-year-study period (year 2000) we invited all FH-NK patients under age 50 who had children and who were registered at the lipid clinic of our institution to take part in the study. Of the $60 \mathrm{FH}-\mathrm{NK}$ patients we found, 41, aged 6-48, agreed to participate. Two patients were excluded because one had a foreign metal body in the eye and the other was claustrophobic. The remaining $39 \mathrm{FH}$ patients formed the patient group. One 45-year-old patient had had a myocardial infarction, and one 46-year-old patient had undergone coronary bypass surgery. The others had no history of diabetes, manifestations of atherosclerosis, or neurological disorders. No signs of intracranial alterations of vascular origin, including infarcts, appeared in their brain MRI (Soljanlahti et al 2005). Eleven patients did not receive statin medication, 9 because of their young age (less than 16), 1 adult did not tolerate statin treatment, and 1 did not use statins regularly. Those 28 patients receiving medication were 27 years old on average at the beginning of statin treatment. At the time of the examination they had, on average, been treated for 10 years.

The control group comprised 13 family members of the patients without the FH-NK mutation and 12 other Finnish controls chosen among acquaintances of our radiology department staff. The controls were 12-50 years old with no history of diabetes, manifestations of atherosclerosis, or neurological disorders. One control was under 15 years of age. In total, 64 subjects, of whom 39 were FH-NK patients and 25 controls, took part (Table 1 ). The study was approved by the ethics committee and research board of our institution, with informed consent obtained from all subjects.

\section{Aortic MRI}

MRI was performed on a $1.5 \mathrm{~T}$ Siemens Vision imager (Erlangen, Germany) with a phased array coil; 23 axial images of the aorta starting from the top of the aortic arch were obtained with a T1-weighted, fat-saturated, turbo spin-echo (TSE) sequence (TR/TE/Flip Angle/FOV/Matrix/ Slice thickness $=2 \mathrm{RR} / 20 \mathrm{~ms} / 90^{\circ} / 200 \times 200 / 128 \times 256 / 6 \mathrm{~mm}$ ) in free breathing. Then a breath hold oblique sagittal TSE sequence (TR/TE/ Flip Angle/FOV/Matrix/Slice Thickness $=700 \mathrm{~ms} / 30 \mathrm{~ms} / 180 / 400 \times 300 / 130 \times 256 / 7 \mathrm{~mm})$

Table I Characteristics and risk factor levels of FH-NK patients and controls

\begin{tabular}{llll}
\hline & FH-NK $\mathbf{~}=\mathbf{3 9}$ & Controls $\mathbf{n}=\mathbf{2 5}$ & P value \\
\hline Age, years & $30.0 \pm 13.6$ & $30.6 \pm 11.3$ & 0.847 \\
BMI, $\mathrm{kg} / \mathrm{m}^{2}$ & $22.3 \pm 4.1$ & $23.0 \pm 2.4$ & 0.443 \\
Systolic blood pressure, $\mathrm{mmHg}$ & $120.9 \pm 14.8$ & $117.9 \pm 11.2$ & 0.402 \\
Diastolic blood pressure, $\mathrm{mmHg}$ & $70.5 \pm 1 \mathrm{I} . \mathrm{I}$ & $69.0 \pm 9.8$ & 0.580 \\
Smokers, number & 7 & 2 & 0.304 \\
Total cholesterol, $\mathrm{mmol} / \mathrm{L}$ & $5.24 \pm 1.70$ & $3.89 \pm 1.03$ & 0.001 \\
$\mathrm{HDL}$ cholesterol, $\mathrm{mmol} / \mathrm{L}$ & $1.13 \pm 0.4 \mathrm{I}$ & $1.19 \pm 0.30$ & 0.282 \\
LDL cholesterol, $\mathrm{mmol} / \mathrm{L}$ & $3.68 \pm 1.41$ & $2.27 \pm 0.82$ & 0.000 \\
Triglycerides, mmol/L & $0.94 \pm 0.48$ & $0.93 \pm 0.32$ & 0.736 \\
Cholesterol-years, mmol-y/L & $243 \pm 122$ & $137 \pm 74$ & 0.001 \\
\hline
\end{tabular}

Values are mean (SD).

Abbreviations: FH, familial hypercholesterolemia-North Karelia; BMI, body mass index; HDL, high density lipoprotein; LDL, low density lipoprotein. 
was taken to assess the imaging plane on the level of the right pulmonary artery for the velocity-encoded flow-measurement sequence (TR/TE/Flip Angle/FOV/Matrix/Slice Thickness/ $\mathrm{VENC}=26 \mathrm{~ms} / 5 \mathrm{~ms} / 30^{\circ} / 300 \times 300 / 256 \times 256 / 6 \mathrm{~mm} / 150$ $\mathrm{cm} / \mathrm{s})$. ECG gating was used with all sequences.

The images were analyzed by 2 reviewers blinded to the clinical and laboratory data. Two morphologic (lumen and wall volume) and 2 functional measures (pulse wave velocity [PWV], and compliance) were assessed. The T1-weighted images starting from the first slice below left arteria subclavia and ending at the last one above the celiac trunk were analyzed if they had sufficient contrast to allow the visual definition of $>50 \%$ of the circumference of the aortic wall. The subject was excluded from analysis if $\geq 50 \%$ of the slices did not meet these criteria. In total, the T1-TSE data of 53 subjects (30 patients and 23 controls) were analyzed. First, the boundaries of the lumen and outer wall of the descending aorta were determined by manual tracing with MRIcro software (author: Chris Rorden, University of South Carolina, Columbia, SC, USA). The area of the lumen and wall on each slice was assessed and their volumes were calculated by multiplying the area by the sum of slice thickness $(6 \mathrm{~mm})$ and slice gap $(6 \mathrm{~mm})$. These volumes were added to provide the entire volume of the aortic wall and lumen. The values were adjusted by the body surface area $(\mathrm{BSA})=[(\text { height }(\mathrm{cm}) * \text { weight }(\mathrm{kg})) / 3600]^{1 / 2}$ of the patient. The velocity-encoded flow images were analyzed with NIH Image software (National Institutes of Health, Bethesda, MD, USA). The mean velocity of the blood flow in the ascending (AA) and descending aorta (DA) was assessed as a function of time. The temporal resolution was $26 \mathrm{~ms}$. The pulse wave was considered to arrive when the velocity reached half of its maximum. From the velocity - time curves, the time delay of the pulse wave arrival between the ascending and descending aorta was measured. The distance between the two measurement sites was assessed from the oblique sagittal image as the mean of the distances along the inner and outer rims of the aorta. The PWV was calculated by dividing the distance by the delay in the pulse wave arrival between the measuring sites (Mohiaddin et al 1993). Compliance (\%/ $\mathrm{mmHg}$ ) was calculated from the anatomical set of flow images by the equation: [(Amax-Amin)/Amin*100]/(Ps-Pd), where Amax $=$ maximum luminal area, Amin $=$ minimal luminal area, $\mathrm{Ps}=$ systolic blood pressure, and $\mathrm{Pd}=$ diastolic blood pressure (Mohiaddin et al 1989).

\section{Carotid US}

All US examinations were performed with a Siemens Acuson 128 XP/10 imager (Erlangen, Germany) and a 7 Mhz linear transducer by the same radiologist. Subjects were examined in supine position with the head turned slightly to the right. The IMT of the far wall of the left CCA was measured, in high-resolution B-mode (Pignoli et al 1986). The mean of 3 consecutive measurements was calculated. CCA strain (\%) was calculated by the equation: maximal diameter of CCA minus minimal diameter of CCA, and this was divided by the minimal diameter of CCA multiplied by 100 (Pearson et al 1996). The mean was calculated for 5 consecutive measurements.

\section{Laboratory tests}

After overnight fasting, each subject gave a venous blood sample. Serum total cholesterol, high-density lipoprotein (HDL) cholesterol, and triglycerides were determined by standard methods. The low-density lipoprotein (LDL) cholesterol value was calculated by the Friedewald formula (Friedewald et al 1972). To take into account the subjects' lifelong cholesterol burden, we calculated the cholesterolyears score, as suggested by Hoeg et al (1994). A DNA test (Koivisto et al 1993) was performed on all FH-NK patients and controls to detect the LDL receptor mutation. Blood pressure was measured twice from the left arm of each subject lying supine by means of an automatic Omega $1400^{\mathrm{TM}}$ meter (Invivo Research Inc, Orlando, FL, USA). The mean of 2 measurements was used in calculations.

\section{Statistical methods}

The means were compared with t-test or Mann-Whitney U-test, as appropriate. Categorial variables were compared with Fisher's exact test. Correlations were calculated with Pearson's or Spearman's test. Multiple regression analysis was used to assess the factors best explaining changes in the functional and morphologic variables of the aorta. Age, gender, BMI, systolic RR, diastolic RR, LDL cholesterol, cholesterol-years score, and FH disease were included in the model. For lumen and wall volume measures, intraclass correlation coefficient was assessed to evaluate inter rater reliability. The analyses were performed with NCSS 2000 program (NCSS, Kaysville, UT, USA) and SPSS 13.0 (SPSS inc., Chicago, IL, USA). P value $<0.05$ was considered statistically significant and used as a cut-off value in multiple regression analysis.

\section{Results}

No statistical difference appeared for any of the aortic measures (BSA-adjusted volume of the aortic lumen and wall, PWV, or the compliance of AA and DA) between FH-NK 
patients and controls (Table 2). Age, BMI, blood pressure, HDL cholesterol level, and cholesterol-years score correlated significantly with almost all aortic measures (Table 3). The independent explaining factors for the variation in aortic measures assessed with multiple regression analysis were age or gender or both for all measures except for the compliance of the DA (Table 4). All the aortic measures correlated significantly with each other except compliance of the DA and volume of the aortic wall (Table 5). Intraclass correlation coefficient was 0.979 and 0.523 for lumen and wall volume, respectively.

The mean IMT of the far wall of the CCA was significantly greater in the FH-NK patients than in the controls (Table 2). The strain of the CCA showed no significant difference between patients and controls (Table 2). Significant correlations appeared between carotid (IMT, CCA strain) and aortic measures except between IMT and compliance of DA (Table 5).

We found no statistical difference in BMI, blood pressure, HDL cholesterol, or triglycerides between patients and controls (Table 1), while patients had higher total and LDL cholesterol levels and cholesterol-years score.

\section{Discussion}

We found no difference in the aortic MRI measures (BSAadjusted volume of the aortic lumen and wall, PWV, or the compliance) between the FH-NK patients and controls despite patients' significantly greater cholesterol-years score and carotid IMT. In the early 1990s when statin medication had been in use only for a short time, 30\% of Finnish FHNK patients over age 25 had CHD (Vuorio et al 1997). In our study the lack of differences in aortic MRI between the groups may be due to the positive effect of improved risk factor treatment, statin medication in particular. Statin treatment induces regression of atherosclerotic lesions, which has been seen in MRI of aorta and carotid arteries (Corti et al 2002) and in US of carotid arteries (Smilde et al 2001).

In our study no difference existed between study groups in morphologic measurements of the aorta (volume of wall and lumen). Eleven percent regression of the aortic and carotid wall area was observed with MRI in 21 asymptomatic hypercholesterolemic patients after 12 months of simvastatin therapy (Corti et al 2002). In another MRI study, $18 \%$ regression of wall area of thoracic aorta was observed in 19 asymptomatic hypercholesterolemic patients after 12 months of atorvastatin therapy (Yonemura et al 2005). Jaffer et al (2002) measured aortic wall area and found out that $38 \%$ of women and $41 \%$ of men free of clinically apparent CHD (aged 36-78) had evidence of subclinical aortic atherosclerosis.

In our study the compliance in aortic MRI and strain of CCA observed with US showed that the arterial walls of the patients were more compliant (although not significantly) than those of the controls. The aortic PWV, however, showed no difference between the groups. Earlier MRI studies have demonstrated that in atherosclerosis, along with morphologic changes, the arterial walls stiffen (Mohiaddin et al 1989; Forbat et al 1998). Some evidence exists, however, that in the early stages of atherosclerosis, the accumulation of lipids makes the arterial wall more compliant before the occurrence of fibrosis (Lehmann et al 1992; Kupari et al 1994). Furthermore, Toikka et al (1999) saw no difference between arterial distensibility assessed with aortic MRI and carotid US of 10 asymptomatic FH patients and controls aged 34 years on average.

We found positive correlations between PWV and BSAadjusted volume of the aortic wall. Correlations between these measures and compliance were negative, indicating that all our MRI parameters were consistent in measuring aortic properties (Table 5). The lumen did not narrow when

Table 2 Morphologic and functional measures of aorta and left common carotid artery of FH-NK patients and controls

\begin{tabular}{llll}
\hline & FH-NK $\mathbf{n}=\mathbf{3 9}$ & Control $\mathbf{n}=\mathbf{2 5}$ & P value \\
\hline Volume of aortic wall/BSA, $\mathrm{mm}^{3} / \mathrm{m}^{2}$ & $10563 \pm 1826$ & $10200 \pm 1358$ & 0.427 \\
Volume of aortic lumen/BSA, $\mathrm{mm}^{3} / \mathrm{m}^{2}$ & $29252 \pm 6865$ & $29063 \pm 564 \mid$ & 0.915 \\
Pulse wave velocity, $\mathrm{m} / \mathrm{s}$ & $3.86 \pm 0.85$ & $3.87 \pm 0.82$ & 0.964 \\
Compliance AA, \%/mmHg & $0.76 \pm 0.39$ & $0.64 \pm 0.29$ & 0.237 \\
Compliance DA, \%/mmHg & $0.57 \pm 0.21$ & $0.47 \pm 0.13$ & 0.062 \\
Left CCA IMT, mm & $0.57 \pm 0.13$ & $0.48 \pm 0.13$ & 0.003 \\
CCA strain, \% & $15.18 \pm 6.92$ & $12.95 \pm 3.33$ & 0.502 \\
\hline
\end{tabular}

Values are mean (SD).

Abbreviations: FH-NK, familial hypercholesterolemia-North Karelia; BSA, body surface area; AA, ascending aorta; DA, descending aorta; CCA, common carotid artery; IMT, intima-media thickness.

Compliance $=[($ systolic area - diastolic area $) /$ diastolic area $* 100] /($ systolic BP-diastolic BP). CCA strain $=($ systolic diameter of CCA - diastolic diameter of CCA $) /$ diastolic diameter of CCA $* 100$. 


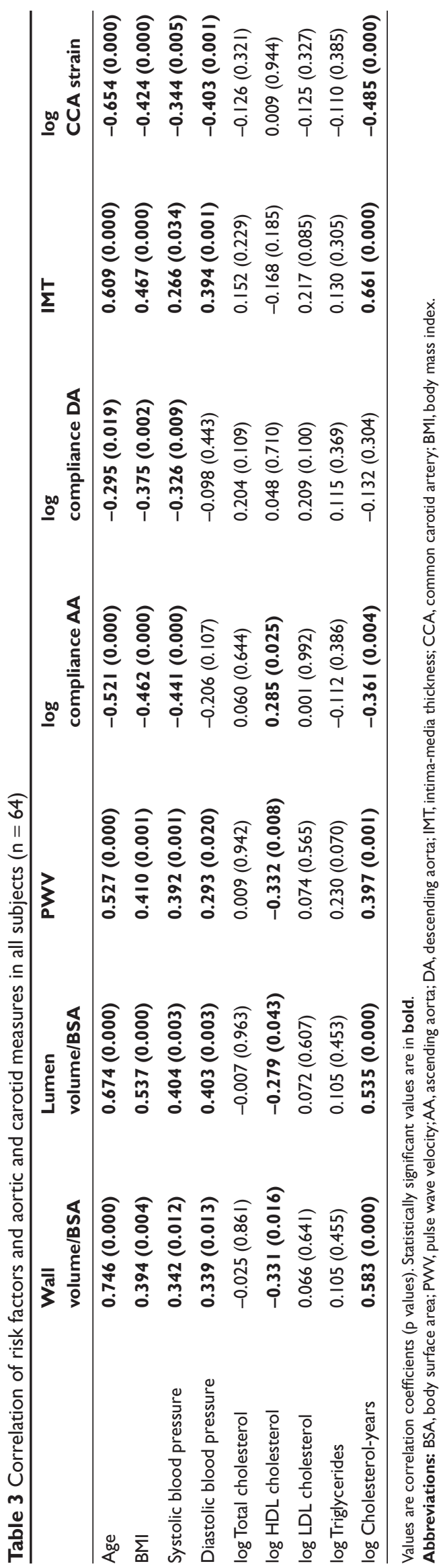

the wall became thicker and stiffer, which could be a sign of positive remodeling (Glagov et al 1987) in our symptomless and relatively young patients.

In our study, established risk factors for atherosclerosis correlated significantly with almost all the aortic measures (Table 3). The compliance of the ascending aorta correlated with more risk factors than compliance of the descending aorta. As DA is smaller than AA and distends less, the change in area between systolic and diastolic images are more difficult to quantify at the spatial resolution of $1.2 \mathrm{~mm} * 1.2 \mathrm{~mm}$.

Age and gender explained best the variation in aortic measures in our study (Table 4) and in the literature (Li et al 2004).

The major limitation of our study is that we could not get an age- and sex-matched control group. Also, not all the FH patients received statin therapy. The wide age range and statin therapy was taken into account by using cholesterol-years score, a parameter that correlated significantly with almost all aortic measures (Table 3). Secondly, we believe that the statin therapy would normalize the aortic MRI parameters of the FH group even further, since the young untreated FH patients represent a subgroup with increased cholesterol year score. Thirdly, age was found to be an independent explaining factor for the variation in aortic measures assessed with multiple regression analysis, a result only found in a group with a wide range of age. In this FH patient group with uniform NK mutation the aortic MRI parameters we used appeared less sensitive than carotid IMT in indicating subclinical atherosclerosis. However, similar parameters are able to show morphologic and functional changes in aortic atherosclerosis (Chan et al 2001; Metafratzi et al 2002; Li et al 2004). Our data are in concordance with a study on Framingham Risk Strata which shows low correlation between aortic MRI and carotid IMT in detection of subclinical atherosclerosis (Kathiresan et al 2007).

In conclusion, using several MRI methods we studied aortic properties in a group of young $\mathrm{FH}$ patients with a uniform FH-NK mutation. Morphologic and functional parameters were comparable between FH-NK patients and controls. The patients had increased carotid IMT in US, showed positive remodeling and a trend to decreased stiffness in the aortic MRI. Morphologic and functional MRI parameters correlated consistently with each other and with cholesterol burden, which accentuates the results. The improved care of cardiovascular risk factors may protect the aorta of FH patients. Another possible explanation for the lack of difference is that aortic MRI may be less sensitive in finding subclinical 
Table 4 Variables explaining variation in aortic MRI measures in all subjects $(n=64)$ assessed with multiple regression analyses

\begin{tabular}{lllll}
\hline Variable & $\begin{array}{l}\text { Explanatory } \\
\text { variable(s) }\end{array}$ & $\begin{array}{l}\text { Regression } \\
\text { coefficient }\end{array}$ & $\mathbf{9 5 \%} \mathbf{C l}$ & $\begin{array}{l}\text { Adjusted } \\
\mathbf{R}^{\mathbf{2}}\end{array}$ \\
\hline Volume of aortic wall/BSA & Age & 103.8 & 0.546 \\
Volume of aortic lumen/BSA & Gender & -3934.1 & $77.4-130.2$ & 0.560 \\
& Age & 386.1 & $-6292.1-(-1576)$ & $284.4-487.8$ \\
Pulse wave velocity & Gender & -0.476 & $-0.816-(-0.135)$ & 0.357 \\
Compliance AA & Age & 0.036 & $0.022-0.049$ & $0.100-0.263$ \\
Compliance DA & Gender & 0.182 & $-0.013-(-0.006)$ & 0.453 \\
\hline
\end{tabular}

Abbreviations: BSA, Body surface area; AA, Ascending aorta; Cl, confidence interval; DA, descending aorta; BMI, Body mass index.

Table 5 Correlation of aortic and carotid measures in all subjects $(n=64)$

\begin{tabular}{lllllll}
\hline & $\begin{array}{l}\text { Lumen } \\
\text { volume/BSA }\end{array}$ & PWV & $\begin{array}{l}\text { log } \\
\text { compliance AA }\end{array}$ & $\begin{array}{l}\text { log } \\
\text { compliance DA }\end{array}$ & $\begin{array}{l}\text { IMT } \\
\text { Log } \\
\text { CCA strain }\end{array}$ \\
\hline Wall volume/BSA & $\mathbf{0 . 8 8 5 ( 0 . 0 0 0 )}$ & $\mathbf{0 . 4 2 5 ( 0 . 0 0 2 )}$ & $-0.499(0.000)$ & $-0.231(0.100)$ & $\mathbf{0 . 3 6 0 ( 0 . 0 0 8 )}$ & $-0.547(0.000)$ \\
Lumen volume/BSA & & $\mathbf{0 . 4 2 2 ( 0 . 0 0 2 )}$ & $-0.589(0.000)$ & $-0.353(0.010)$ & $0.337(0.014)$ & $-0.534(0.000)$ \\
PWV & & & $-0.685(0.000)$ & $-0.318(0.011)$ & $0.307(0.015)$ & $-0.354(0.004)$ \\
log Compliance AA & & & & $0.465(0.000)$ & $-0.327(0.009)$ & $0.404(0.001)$ \\
log Compliance DA & & & & $-0.142(0.266)$ & $0.281(0.026)$ \\
IMT & & & & & $-0.277(0.027)$ \\
\hline
\end{tabular}

Values are correlation coefficients with $p$ values in brackets. Statistically significant values are in bold.

Abbreviations: BSA, body surface area; PWV, pulse wave velocity; AA, ascending aorta; DA, descending aorta; IMT, intima-media thickness; CCA, common carotid artery.

atherosclerosis than carotid US. Larger studies are needed to confirm these hypotheses and to obtain normal values for aortic morphology and function. The influence of age and gender on vascular properties was marked and should be taken into account in further studies.

\section{Acknowledgments}

The authors wish to thank the FH-NK patients, the controls, and Timo Päivärinta and Aki Syrjälä for technical assistance. This study was supported by grants from the Radiological Society of Finland, the Paulo and P.O Klingendahl Foundations, Schering research foundation and Finnish Cultural Foundation and the Helsinki University Central Hospital Research Funds (TYH 1216, TYH 3229).

\section{Disclosures}

None of the authors has any financial or other relationships that might lead to a conflict of interest.

\section{References}

Anonymous. 1999. Mortality in treated heterozygous familial hypercholesterolaemia: implications for clinical management. Scientific Steering Committee on behalf of the Simon Broome Register Group. Atherosclerosis, 142:105-12.
Chan S, Jaffer F, Botnar R, et al. 2001. Scan reproducibility of magnetic resonance imaging assessment of aortic atherosclerosis burden. $J$ Cardivasc Magn Res, 3:331-8.

Clarke SE, Hammond RR, Mitchell JR, et al. 2003. Quantitative assessment of carotid plaque composition using multicontrast MRI and registered histology. Magn Reson Med, 50:1199-208.

Corti R, Fuster V, Fayad ZA, et al. 2002. Lipid lowering by simvastatin induces regression of human atherosclerotic lesions: Two years' follow-up by high-resolution noninvasive magnetic resonance imaging. Circulation, 106:2884-7.

Forbat SM, Naoumova RP, Sidhu PS, et al. 1998. The effect of cholesterol reduction with fluvastatin on aortic compliance, coronary calcification and carotid intimal-medial thickness: a pilot study. $J$ Cardiovasc Risk, 5:1-10.

Friedewald WT, Levy RI, Fredrickson DS. 1972. Estimation of the concentration of low-density lipoprotein cholesterol in plasma, without use of the preparative ultracentrifuge. Clin Chem, 18:499-502.

Glagov S, Weisenberg E, Zarins CK, et al.1987. Compensatory enlargement of human atherosclerotic coronary arteries. $N$ Engl J Med, 316:1371-5.

Goldstein JL, Hobbs HH, Brown MS. 2001. Familial hypercholesterolemia. In: Scriver CR, Beaudet AL, Sly WS, editors. The metabolic and molecular bases of inherited diseases. New York: McGraw-Hill Book Co. pp. 2863-913.

Hoeg JM, Feuerstein IM, Tucker EE. 1994. Detection and quantitation of calcific atherosclerosis by ultrafast computed tomography in children and young adults with homozygous familial hypercholesterolemia. Arterioscler Thromb, 14:1066-74.

Jaffer FA, O’Donnell CJ, Larson MG, et al. 2002. Age and sex distribution of subclinical aortic atherosclerosis: a magnetic resonance imaging examination of the Framingham Heart Study. Arterioscler Thromb Vasc Biol, 22:849-54. 
Kathiresan S, Larson M, Keyes M, et al. 2007. Assessment by cardiovascular magnetic resonance, electron beam computed tomography, and carotid ultrasonography of the distribution of subclinical atherosclerosis across Framingham risk strata. Am J Cardiol, 99:310-4.

Koivisto U-M, Hamalainen L, Taskinen MR, et al. 1993. Prevalence of familial hypercholesterolemia among young north Karelian patients with coronary heart disease: a study based on diagnosis by polymerase chain reaction. J Lipid Res, 34:269-77.

Koivisto U-M, Turtola H, Aalto-Setälä K, et al. 1992. The familial hypercholesterolemia (FH)-North Karelia mutation of the low density lipoprotein receptor gene deletes seven nucleotides of exon 6 and is a common cause of FH in Finland. J Clin Invest, 90:219-28.

Kupari M, Hekali P, Keto P, et al. 1994. Relation of aortic stiffness to factors modifying the risk of atherosclerosis in healthy people. Arterioscler Thromb, 14:386-94.

Lehmann ED, Watts GF, Fatemi-Langroudi B, et al. 1992. Aortic compliance in young patients with heterozygous familial hypercholesterolaemia. Clin Sci, 83:717-21.

Li AE, Kamel I, Rando F, et al. 2004. Using MRI to assess aortic wall thickness in the multiethnic study of atherosclerosis: distribution by race, sex, and age. Am J Roentgenol, 182:593-7.

Metafratzi Z, Efremidis S, Skopelitou A, et al. 2002. The clinical significance of aortic compliance and its assessment with Magnetic resonance imaging. J Cardiovasc Magn Res, 4:481-91.

Mohiaddin RH, Underwood SR, Bogren HG, et al. 1989. Regional aortic compliance studied by magnetic resonance imaging: the effects of age, training, and coronary artery disease. Br Heart J, 62:90-6.

Mohiaddin RH, Firmin DN, Longmore DB. 1993. Age-related changes of human aortic flow wave velocity measured noninvasively by magnetic resonance imaging. J Appl Physiol, 74:492-7.

Oliver JJ, Webb DJ. 2003. Noninvasive assessment of arterial stiffness and risk of atherosclerotic events. Arterioscler Thromb Vasc Biol, 23:554-66.
Pearson AC, Peterson JW, Orsinelli DA, et al.1996. Comparison of thickness and distensibility in the carotid artery and descending thoracic aorta: in vivo ultrasound assessment. Am Heart J, 131:655-62.

Pignoli P, Tremoli E, Poli A, et al. 1986. Intimal plus medial thickness of the arterial wall: a direct measurement with ultrasound imaging Circulation, 74:1399-406.

Reunanen A, Kattainen A; Working group for cardiovascular diseases. 2004. Cardiovascular diseases and diabetes. In: Aromaa A, Koskinen $\mathrm{S}$ editors. Health and functional capacity in Finland: baseline results of the Health 2000 health examination survey. Helsinki: National Public Health Institute, Finland. pp. 47-51.

Smilde TJ, van Wissen S, Wollersheim H, et al. 2001. Effect of aggressive versus conventional lipid lowering on atherosclerosis progression in familial hypercholesterolaemia (ASAP): a prospective, randomised, double-blind trial. Lancet, 357:577-81.

Soljanlahti S, Autti T, Lauerma K, et al. 1995. Familial hypercholesterolemia patients treated with statins at no increased risk for intracranial vascular lesions despite increased cholesterol burden and extracranial atherosclerosis. Stroke, 36:1572-4.

Sternby NH. 1968. Atherosclerosis in a defined population: an autopsy survey in Malmö, Sweden. Acta Pathol Microbiol Scand, 194(Suppl):3-216.

Toikka JO, Niemi P, Ahotupa M, et al. 1999. Large-artery elastic properties in young men: relationships to serum lipoproteins and oxidized lowdensity lipoproteins. Arterioscler Thromb Vasc Biol, 19:436-41.

Vuorio AF, Turtola H, Piilahti KM, et al. 1997. Familial hypercholesterolemia in the Finnish north Karelia. A molecular, clinical, and genealogical study. Arterioscler Thromb Vasc Biol, 17:3127-38.

Yonemura A, Momiyama Y, Fayad ZA, et al. 2005. Effect of lipid-lowering therapy with atorvastatin on atherosclerotic plaques detected by noninvasive magnetic resonance imaging. J Am Coll Cardiol, 45:733-42.

Yuan C, Kerwin WS. 2004. MRI of atherosclerosis. J Magn Reson Imaging, 19:710-9. 
